\title{
Requerimentos para uso de tratamentoshormonaise desmame temporário no controle da reprodução de vacas de corte com cria ao pé
}

\author{
Requirements for the use of hormonal treatments and temporary weaning to control reproduction \\ in suckling beef cows
}

\author{
José Carlos Ferrugem Moraes ${ }^{1}$, Carlos J osé Hoff de Souza ${ }^{1} \&$ Carlos Miguel Jaume ${ }^{2}$
}

\begin{abstract}
RESUMO
A busca de alternativas locais para incremento da fertilidade em vacas de corte com cria ao pé tem sido efetuada através de estudos da fisiologia do pós-parto e do desenvolvimento de sistemas de indução de ovulação e cio durante o pósparto precoce. Embora seja possível a indução de ovulação durante o pós-parto, o balanço energético negativo nos primeiros meses após o parto, associado à amamentação e à carência de alimento nesse período são os principais fatores determinantes da baixa fertilidade de vacas de corte criadas sob condições extensivas. O objetivo foi o de identificar a condição corporal mínima para a utilização de tratamentos hormonais associados a desmame temporário em condições específicas de criação para a melhoria dos índices de reprodução de vacas com cria ao pé. Um total de 904 vacas oriundas de cruzamentos comerciais criadas em duas propriedades foram submetidas à inseminação e monta natural subsequente com duração de até 90 dias após a aplicação de separação dos terneiros por 96 horas, suplementação durante sete dias com progestágenos via intravaginal seguida ou não de uma injeção de estrógeno após 24 horas. As taxas de inseminação e prenhez foram analisadas através de logaritmo linear, considerando possíveis interações entre os fatores: condição corporal, propriedade, grupo de parição e tratamentos hormonais. Os resultados indicaram que apenas vacas em escore superior a 3 numa escala de 1 a 5 devem receber tratamento hormonal, devendo ser consideradas as interações entre os tratamentos e grupo de parição, bem como os efeitos diferenciais em cada unidade de produção.
\end{abstract}

Descritores: bovinos, fertilidade pós-parto, condição corporal, data dos partos.

\section{ABSTRACT}

The search of suitable local systems to improve fertility rates in beef cattle have been made through studies on postpartum physiology and the development of systems to induce ovulation and estrus early in post-partum. Notwithstanding ovulation and estrous could be achieved, the negative energetic balance in the first months after calving associated with suckling and forage availability were the determinant factors of low fertility in extensive systems. The aim was to identify the low body condition score of the cows in which they could be submit to treatments with a progestagen associated to temporary weaning in specific farm conditions in order to improve fertility indices in suckling cows. A total of 904 beef cows, derived from commercial crosses and raised in two private farms, were submitted to artificial insemination followed by natural mating up to 90 days, after temporary weaning of 96 hours, intra-vaginal supplementation of a progestagen followed or not by an injection of estradiol 24 hours after. The frequencies of inseminated and pregnant cows were analyzed by log-lin procedures considering the possible interactions among: body condition scores, farm, calving groups and hormonal treatments. The observed results indicates that just cows with body condition score higher than 3 (range 1 to 5) should be treated with the progestagen, considering the interactions between calving groups and hormonal treatments in each farm.

Keywords: bovine, post-partum fertility, body condition score, calving date. 


\section{INTRODUÇÃO}

O balanço energético negativo após o parto é incontestável em vacas de leite e de corte [e.g., 2,10], podendo ser evidenciado pela redução e variação da condição corporal durante o pós-parto [8,9]. Assim, a deficiente condição corporal no início do período de reprodução das vacas com cria ao pé [e.g. 1], associada à época do ano, data dos partos, raça, idade, paridade, amamentação, presença de machos e suas interações com a disponibilidade de forragem resulta no anestro pós-parto [12] determinante das baixas taxas de fertilidade das vacas com cria ao pé, no Rio Grande do Sul, na ordem de 50-60\% [11].

Diversos sistemas de tratamentos hormonais são capazes de induzir ovulação e cio durante o anestro pós-parto em vacas de corte [e.g. 4]. Entretanto esses procedimentos não apresentam os resultados esperados, quando utilizados em animais em pobre CC e durante pós-parto precoce.

No contexto do controle da reprodução, é importante definir quais os requerimentos mínimos para o uso de tratamentos à base de gestágenos em vacas de corte mantidas em sistemas extensivos de criação, considerando períodos de reprodução em torno de 90 dias e a pobre condição corporal das vacas paridas durante o final do inverno - início da primavera no sul do Brasil. Assim, o objetivo deste estudo é investigar a condição corporal mais adequada para a reprodução de vacas com crias ao pé mantidas em dois sistemas de criação, empregando tratamentos hormonais associados a desmame temporário.

\section{MATERIAIS E MÉTODOS}

Novecentos e quatro vacas de corte amamentando suas crias, mantidas sobre pastagem nativa na região da Campanha do Rio Grande do Sul, em duas propriedades particulares de um único produtor (A: localizada em Bagé $\left(31^{\circ} 04^{\prime} \mathrm{N}, 54^{\circ} 00^{\prime} \mathrm{O}\right)$ e B: localizada em Pedras Altas $\left(31^{\circ} 45^{\prime} \mathrm{N}, 5^{\circ} 35^{\prime} \mathrm{O}\right)$ foram estratificadas quanto a data de seus partos em quatro grupos de três semanas cada um. Esse procedimento permitiu o início do período de reprodução das vacas entre 63-84 dias pós-parto (dpp). Na Tabela 1, são apresentadas as datas limites em que foram formados os grupos de parição e o efetivo início dos procedimentos reprodutivos nas duas propriedades onde foram implementados os experimentos.

$\mathrm{Na}$ data de início dos procedimentos, cada grupo de vacas foi distribuído ao acaso, em três tratamentos distintos: Testemunha, incluindo vacas amamentando suas crias mantidas em conjunto com as demais, as vacas deste lote foram separadas de suas crias apenas quando expostas à inseminação artificial; MAP, incluindo vacas que receberam, na data de início dos procedimentos, um pessário contendo $250 \mathrm{mg}$ de acetato de medroxi-progesterona e $5 \mathrm{mg}$ de benzoato de estradiol via intramuscular, na data de início da cobrição, os pessários foram removidos, bem como as crias por um período de 96 horas, durante o qual as vacas foram monitoradas quanto à manifestação de estro, sendo aquelas detectadas em cio inseminadas 12 horas após; e, MAP+ODB 24 horas, incluindo vacas que foram submetidas ao mesmo procedimento descrito para o tratamento MAP, mais uma injeção de 0,5 mg de benzoato de estradiol 24 horas após a remo-ção os pessários. O número total de animais em cada grupo e tratamento nas duas propriedades está apresentado na Tabela 2. Adicionalmente, no momento de início dos procedimentos, todas as vacas foram avaliadas quando a sua condição corporal, empregando o sistema de escores de cinco classes [13] preconizado pela EMATER,

Tabela 1. Datas utilizadas para a formação dos grupos de parição e procedimentos de controle da reprodução nas duas propriedades particulares localizadas nos municípios de Bagé e Pedras Altas.

\begin{tabular}{ccccccc}
\hline & \multicolumn{3}{c}{ Propriedade A* } & \multicolumn{3}{c}{ Propriedade B\# } \\
\hline Grupo & $\begin{array}{c}\text { Final } \\
\text { parição }\end{array}$ & $\begin{array}{c}\text { Início } \\
\text { procedimentos }\end{array}$ & $\begin{array}{c}\text { Início } \\
\text { cobrição }\end{array}$ & $\begin{array}{c}\text { Final } \\
\text { parição }\end{array}$ & $\begin{array}{c}\text { Início } \\
\text { procedimentos }\end{array}$ & $\begin{array}{c}\text { Início } \\
\text { cobrição }\end{array}$ \\
\hline 1 & $04 / 09$ & $23 / 10$ & $30 / 10$ & $10 / 09$ & $29 / 10$ & $05 / 11$ \\
2 & $25 / 09$ & $13 / 11$ & $20 / 11$ & $01 / 10$ & $19 / 11$ & $16 / 11$ \\
3 & $16 / 10$ & $04 / 12$ & $11 / 12$ & $22 / 10$ & $10 / 12$ & $17 / 12$ \\
4 & $06 / 11$ & $26 / 12$ & $03 / 01$ & $12 / 11$ & $31 / 12$ & $07 / 01$ \\
\hline
\end{tabular}

* Final do período reprodutivo de 90 dias em 28/01; início previsto dos partos 05/08. \# Final do período reprodutivo de 90 dias em 03/02; início previsto dos partos 11/08. 
no Rio Grande do Sul [3]. Após a conclusão das observações de cio, durante os quatro dias, as vacas continuaram a amamentar suas crias e foram submetidas à monta natural com $2 \%$ de touros previamente avaliados quanto à sua potencialidade reprodutiva.

As variáveis medidas foram a percentagem de vacas inseminadas nos quatro dias de controle de cios e a percentagem total de vacas gestantes, definida por palpação retal, em torno de 60 dias após a conclusão do período reprodutivo, as quais foram submetidas à análise não paramétrica de logaritmo linear, incluindo os fatores CC (2, 3 e 4), Propriedade (A e B), Grupo de parição (1, 2, 3 e 4) e Tratamento (Testemunha, MAP e MAP+ODB24h), empregando o pacote NCSS com a opção de seleção do melhor modelo para explicar a variação observada [6]. Com o objetivo de permitir a análise de dados com frequência zero, foi adicionado e testado um valor delta de 0,1 .

\section{RESULTADOS}

A frequência de vacas com cria ao pé inseminadas com o emprego do sistema preconizado foi afetada pela condição corporal das vacas, pelo siste- ma de produção de cada propriedade e pela interação entre grupo de parição e tratamento administrado ( $\chi 2=10,6 ; 3$ GL; P=0,0139).

No geral, a taxa de cio foi de $32 \%$ (101/314), em vacas em CC2, de 45\% (231/518), em vacas em $\mathrm{CC} 3$ e de $61 \%$ (43/71) nas vacas em CC4 ( $\chi 2=23,9 ; 2$ GL; $\mathrm{P}<0,001)$. No que diz respeito aos sistemas de criação, a frequência de cios foi de $44 \%$ (218/499) na propriedade A e de 39\% (157/405), na propriedade B. Na Tabela 3, são apresentadas as taxas de IA observadas na interação significativa entre grupo de parição e tratamento.

A frequência de vacas prenhas também apenas foi afetada significativamente por interações de segunda ordem entre os fatores avaliados $(\chi 2=138,18 ; 63 \mathrm{GL}$; $\mathrm{P}<0,001)$, tendo sido identificado como melhor modelo o que inclui as interações entre os fatores Grupo de parição x Tratamento, Grupo de parição x CC, Propriedade x CC e ainda Propriedade x Grupo de parição $(\chi 2=30,2 ; 6$ GL; P<0,001). Na Tabela 4, são apresentadas as taxas de prenhez observadas em função dos grupos de parição e tratamento. Na Figura 1, estão apresentadas graficamente as interações significativas

Tabela 2. Número de vacas alocadas nos distintos tratamentos em cada grupo de parição nas duas propriedades particulares localizadas nos municípios de Bagé e Pedras Altas.

\begin{tabular}{cccccccccc}
\hline & \multicolumn{3}{c}{ Prop. } & \multicolumn{2}{c}{ A } & & \multicolumn{3}{c}{ Prop. } \\
\cline { 2 - 4 } \cline { 7 - 9 } Grupo & Test. & MAP & MAP + ODB & Total & & Test. & MAP & MAP + ODB & Total \\
\hline 1 & 54 & 55 & 56 & 165 & & 27 & 29 & 25 & 81 \\
2 & 34 & 64 & 67 & 165 & & 28 & 56 & 63 & 147 \\
3 & 22 & 47 & 50 & 119 & & 20 & 38 & 38 & 96 \\
4 & 9 & 0 & 41 & 50 & & 19 & 0 & 62 & 81 \\
\hline Total & 119 & 166 & 214 & 499 & & 94 & 123 & 188 & 405 \\
\hline
\end{tabular}

Tabela 3. Frequência de vacas inseminadas em quatro dias, submetidas aos três distintos tratamentos, nos quatro grupos de parição.

\begin{tabular}{cccc}
\hline & \multicolumn{3}{c}{ Tratamentos } \\
\cline { 2 - 4 } Grupo de parição & Testemunha & MAP & MAP + ODB 24h \\
& $\mathrm{n} / \mathrm{N}(\%)$ & $\mathrm{n} / \mathrm{N}(\%)$ & $\mathrm{n} / \mathrm{N}(\%)$ \\
\hline 1 & $9 / 81(11)$ & $22 / 84(26)$ & $39 / 81(47)$ \\
2 & $10 / 62(16)$ & $36 / 120(30)$ & $93 / 130(72)$ \\
3 & $13 / 42(31)$ & $31 / 85(36)$ & $71 / 88(81)$ \\
4 & $4 / 28(14)$ & $0 / 0(-)$ & $48 / 103(47)$ \\
\hline
\end{tabular}

n, número de vacas em cio; $\mathrm{N}$, número de vacas em cada grupo de parição e tratamento. 
Tabela 4. Frequência de vacas prenhas, nos distintos grupos de parição, nos tratamentos investigados.

\begin{tabular}{cccc}
\hline & \multicolumn{3}{c}{ Tratamentos } \\
\cline { 2 - 4 } Grupo de parição & Testemunha & MAP & MAP + ODB 24h \\
& n/N (\%) & $\mathrm{n} / \mathrm{N}(\%)$ & $23 / 81(28)$ \\
2 & $29 / 81(36)$ & $33 / 84(39)$ & $53 / 130(41)$ \\
3 & $20 / 62(32)$ & $40 / 120(33)$ & $25 / 88(28)$ \\
4 & $11 / 42(26)$ & $25 / 85(29)$ & $23 / 103(22)$ \\
\hline n, número de vacas prenhas; N, número de vacas em cada grupo de parição e tratamento.
\end{tabular}

quanto a CC, em "a" com Grupo de parição e em "b" com as Propriedades. Sequencialmente, a associação entre Grupo de parição x Propriedade é apresentada na Figura 2.

A dinâmica dos escores de $\mathrm{CC}$ das vacas do início do período reprodutivo (63-84 dias pós-parto) até o diagnóstico de gestação (90-150 dias pós-parto) revelou um padrão semelhante nas duas propriedades. Na Figura 3, é apresentado o percentual de prenhez observado nas distintas classes de variação da CC.

\section{DISCUSSÃO}

O uso da inseminação em vacas com cria ao pé, mesmo com o auxílio de tratamentos hormonais, depende das condições nutricionais das vacas no momento do início do período de reprodução. No caso específico, apenas vacas em $\mathrm{CC} 4$ apresentaram uma taxa de IA superior a $60 \%$, as vacas em CC3 que representavam a maior parte do rebanho, apenas $45 \%$ delas foram inseminadas ainda na dependência do tipo de procedimento utilizado.

As dificuldades observadas e esperadas no uso de práticas de controle da reprodução podem ser explicadas pelas distintas condições de manejo, solo e clima entre propriedades, reiteradas no presente estudo que incluiu animais semelhantes quanto à composição racial e administração.

Quanto às práticas de manejo reprodutivo empregadas fica a indicação de que apenas o desmame por quatro dias, cerca 60 dias pós-parto, não viabiliza uma taxa de inseminação desejável [4]. Além disso, há necessidade de considerar que o uso de tratamentos com gestágeno depende da época dos partos, concentrando para inseminação artificial apenas vacas em CC3 e CC4.

A maior taxa de inseminação foi observada no Grupo de parição 3 (mês de outubro), destacando-se o tratamento que incluiu a medroxi-progesterona associada a $1 \mathrm{mg}$ de benzoato de estradiol, 24 horas após a remoção dos pessários. Muito possivelmente, a associação do estrógeno na remoção do gestágeno tenha facilitado a identificação de cio nas vacas recém desmamadas ou mesmo promovido a maior freqüência de em estro, como já demonstrado com progesterona [5].

A maior variação na taxa de gestação foi observada entre Grupos de parição que receberam a injeção de benzoato de estradiol $24 \mathrm{~h}$ após a remoção dos pessários, incluindo a menor e a maior taxa de prenhez em todos os grupos (Tabela 4). Entretanto, a maior taxa de manifestação e/ou indução de cio observada nesse tratamento não foi acompanhada pelos mesmos índices de fertilidade dos demais tratamentos. Embora alguns estudos [5,7] reiterem um baixo efeito na fertilidade de vacas tratadas com benzoato de estradiol entre 24 e 30 horas após a remoção dos dispositivos intravaginais impregnados com progesterona.

No geral, é possível inferir que a utilização de práticas de manejo ou hormonais, visando à melhoria da eficiência reprodutiva, podem ser muito pouco efetivos, se as vacas não se encontram em boa condição nutricional (escores superiores a CC3) e com seus partos ocorrendo na primeira metade do período de parição. Esta inferência contrasta com estudos que sugerem a utilidade dos dispositivos de progesterona na indução de ovulação em torno de $40 \mathrm{dpp}$ em vacas também estratificadas em grupos de parição [14]. Os presentes resultados reiteram a necessidade de ajustes no momento de início do período reprodutivo em cada propriedade em decorrência da deficiente condição corporal das vacas nos sistemas extensivos locais no início do período de reprodução [9].

Essas recomendações estão relacionadas às superiores taxas de prenhez nos primeiros grupos de 

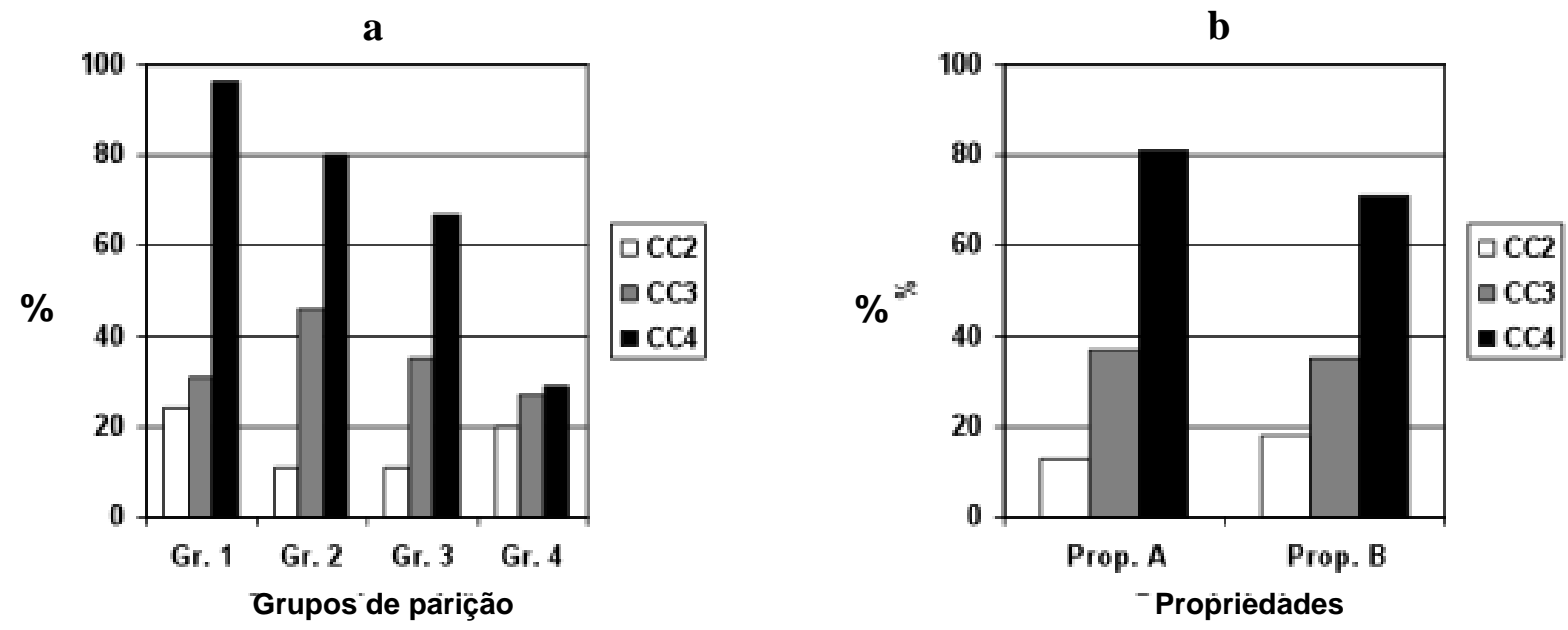

Figura 1. Taxa de gestação nos distintos grupos de parição (a) e propriedades (b) em função dos escores de condição corporal (CC).

parição (Figura 1a) e no fato de que a taxa de prenhez das vacas em escore CC2, apenas supera vinte pontos percentuais naquelas paridas ainda no mês de setembro (Grupo 1). Ainda merece destaque as taxas de gestação de 20\%-30\% para as vacas paridas no mês de novembro (Grupo 4), mesmo em CC3 e CC4. Na Figura 1b, é interessante observar além da consistência das maiores taxas de gestação entre as vacas em $\mathrm{CC} 4$, o fato que as diferenças ambientais e de manejo explicam diferenças na taxa de gestação entre vacas CC2 e CC3, muito possivelmente em relação ao tipo de modificação no nível nutricional do início ao final do período reprodutivo em cada propriedade, que são reiterados pelos contrastes nas taxas de gestação das vacas paridas nos meses de setembro e outubro (Grupos 2 e 3).

A variação dos escores de $\mathrm{CC}$ das vacas do início do período reprodutivo (63-84 dias pós-parto)

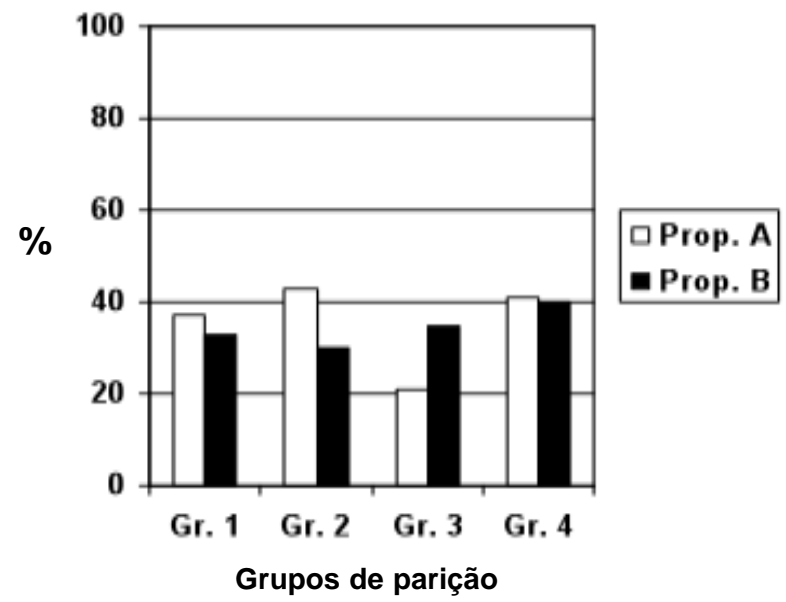

Figura 2. Taxa de gestação nos distintos grupos de parição e propriedades. até o diagnóstico de gestação (90-150 dias pós-parto) sugere que vacas em CC2, mesmo ganhando um escore de $\mathrm{CC}$, ainda apresentam taxa de prenhez insatisfatória. O mesmo se passa com vacas em CC3 que perdem um escore de condição corporal nesse período. Neste contexto, é possível inferir que um melhor desempenho reprodutivo de vacas com cria ao pé já pode ser esperado nas vacas em escore CC 3 , mas ainda depende das condições de criação dessas vacas durante o período de cobrição e início da gestação.

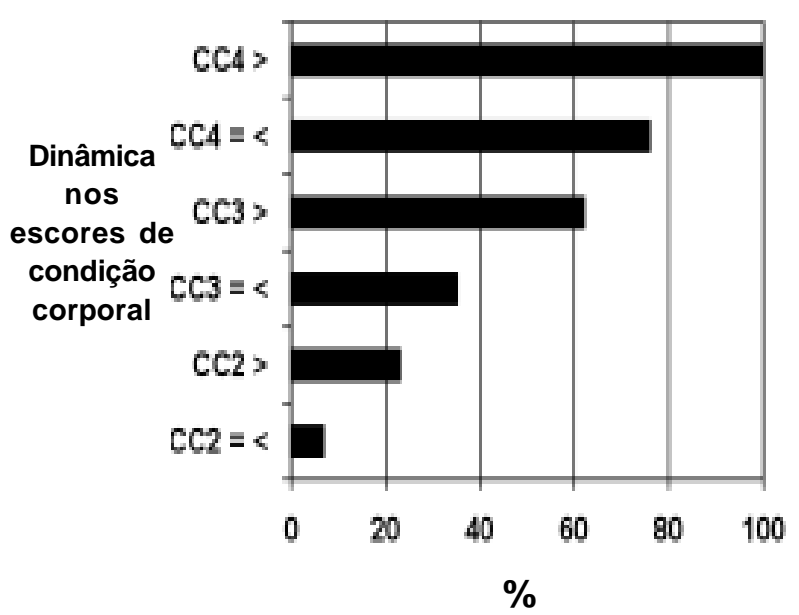

Figura 3. Taxa de gestação em função da dinâmica da condição corporal do início da cobrição até 90-150 dias após. $\mathrm{CC} 2,3$ e $4=<$, representa o percentual de vacas com escore 2 , 3 ou 4 no início do período de cobrição e que mantiveram ou perderam um escore até o diagnóstico de gestação efetuado de 90 a 150 dias depois.

$\mathrm{CC} 2,3$ e 4 >, representa o percentual de vacas com escore 2, 3 ou 4 no início do período de cobrição e que ganharam um escore até o diagnóstico de gestação efetuado de 90 a 150 dias depois. 


\section{CONCLUSÕES}

Nas condições em que foi efetuado o presente estudo, é possível recomendar práticas de controle da reprodução que incluam o uso de tratamentos para a indução de cio e ovulação pós-parto em vacas corte apenas para animais com escore de condição corporal superior a três, considerando uma escala de cinco classes. Além disso, é indispensável o conhecimento da data dos partos, para que sejam efetuados os procedimentos referentes à indução de cios, o que pode ser efetuado através da organização das vacas em lotes de parição. Para a obtenção de maiores taxas de gestação das vacas amamentando as práticas de desmame temporário por 96 horas, devem ser efetuadas após a suplementação com gestágenos. E, ainda como recomendação de ordem geral, os resultados indicam que deve ser proporcionada uma condição de oferta de forragem capaz de promover melhoria na condição corporal das vacas no primeiro terço da gestação.

Agradecimentos. Ao Conselho Nacional de Desenvolvimento Científico e Tecnológico, pelo financiamento parcial e ao produtor rural Sr. José Érico Souto, pela parceria na implementação dos experimentos.

\section{REFERÊNCIAS}

1 Almeida L.S.P., Lobato J.F.P. \& Schenkel F.S. 2002. Data de Desmame e Desempenho Reprodutivo de Vacas de Corte. Revista Brasileira de Zootecnia. 31: 1223-1229.

2 Butler W.R. 2003. Energy balance relationships with follicular development, ovulation and fertility in postpartum dairy cows. Livestock Production Science. 83: 211-218.

3 Cachapuz J.M.A. 1997. Experiências com desmame aos 90 e 60 dias. Porto Alegre: Emater, 52p.

4 Day M.L. 2004. Hormonal induction of estrous cycles in anestrous Bos taurus beef cows. Animal Reproduction Science. 82/83: 487-494.

5 Fike K.E., Day M.L., Inskeep E.K., Kinder J.E., Lewis P.E., Short R.E. \& Hafs H.D. 1997. Estrus and luteal function in suckled beef cows that were anestrous when treated with an intravaginal device containing progesterone with or without a subsequent injection of estradiol benzoate. Journal of Animal Science. 75: 2009-2015.

6 Hintze J. NCSS. Kaysville, Utah. 2006. [www.ncss.com].

7 Lane E.A., Austin E.J., Roche J.F. \& Crowe M.A. 2001. The effect of estradiol benzoate on synchrony of estrus and fertility in cattle after removal of a progesterone releasing intravaginal device. Theriogenology. 55: 1807-1818.

8 Moraes J.C.F., Jaume C.M., Souza C.J.H., Paludo G.R. \& Müller L. 2008. Post-partum follicular dynamics in beef cows calving during spring and autumn in southern Brazil. Communications in Theriogenology, 2: 2002. Available at: <http:/ /ctheriogenology.lsu.edu/ct/Issues/PastIssues/2001-2/2001-2FinalWeb.html>. Acessado em 26 de junho de 2008.

9 Moraes J.C.F., Jaume C.M. \& Souza C.J.H. 2007. Body condition score to predict the postpartum fertility of crossbred beef cows. Pesquisa Agropecuária Brasileira. 42: 741-746.

10 Renquist B.J., Oltjen J.N., Sainz R.D. \& Calvert C.C. 2006. Relationship between body condition score and production of multiparous beef cows. Livestock Science. 104: 147-155.

11 SEBRAE/SENAR/FARSUL. 2005. Diagnóstico de Sistemas de Produção de Bovinocultura de Corte do Estado do Rio Grande do Sul. Relatório. Porto Alegre: IEPE-UFRGS/SENAR, 265p.

12 Short R.E., Bellows R.A., Staigmiller R.B., Berardinelli J.G. \& Custer E.E. 1990. Physiological mechanisms controlling anestrus and infertility in postpartum beef cattle. Journal of Animal Science. 68: 799-816.

13 Wildman E.E., Jones G.M., Wagner P.E. \& Boman R.L. 1982. A dairy cow body condition scoring system and its relationship to selected production characteristics. Journal of Dairy Science. 65: 495-501.

14 Wheaton J.E. \& Lamb G.C. 2007. Induction of cyclicity in postpartum anestrous beef cows using progesterone, GnRH and estradiol cypionate (ECP). Animal Reproduction Science. 102: 208-216. 